\title{
Solving the Solar Neutrino Problem
}

\author{
Ardeshir Irani \\ Dark Energy Research Institute, Downey, USA \\ Email:artirani@aol.com
}

How to cite this paper: Irani, A. (2021) Solving the Solar Neutrino Problem. Journal of High Energy Physics, Gravitation and Cosmology, 7, 1278-1279. https://doi.org/10.4236/jhepgc.2021.74077

Received: July 12, 2021

Accepted: September 5, 2021

Published: September 8, 2021

Copyright (c) 2021 by author(s) and Scientific Research Publishing Inc. This work is licensed under the Creative Commons Attribution International License (CC BY 4.0).

http://creativecommons.org/licenses/by/4.0/

\begin{abstract}
A new theoretical prediction that a plasma can produce antineutrinos is used to solve the solar neutrino problem. The difference between electron-positron induced fusion, and inertial fusion experiments that have been unsuccessful so far as commercial fusion reactors is also discussed.
\end{abstract}

\section{Keywords}

Antineutrinos, Electron-Positron, Fusion Reactors, Solar Neutrino, Plasma

\section{The Plasma as a Source of Antineutrinos}

A plasma contains protons and electrons of equal number. Since they are oppositely charged, they will attract each other and since the proton is 1835 times heavier than the electron the electron will speed up faster to combine with the proton to form a neutron in its excited state. The excitation energy of the neutron comes from the kinetic energy gained by the electron before it forms the neutron. The neutron takes about fifteen minutes to jump back to its normal state according to the following reaction: neutron $=$ proton + electron + antineutrino, and this is the process by which a plasma continuously produces antineutrinos. The nebula [1], the plasma formed by a Supernova explosion can also produce antineutrinos in this fashion as can too the plasma of the solar wind.

\section{The Solar Neutrino Problem Solved}

The solar neutrino problem was regarding a large discrepancy between the flux of solar neutrinos as predicted from the Sun's luminosity and those measured directly. When neutrino detectors became sensitive enough to measure the flow of electron neutrinos from the Sun, the number detected was much lower than that which was predicted. In various experiments, the number deficit was between one half and two thirds. While neutrino oscillations between electron, muon, and tau neutrinos could be used as an explanation for the deficit in elec- 
tron neutrinos it is clear that is not what was happening because if that were the case then the sum of all the three neutrinos should be equal to the expected value of electron neutrinos from the Sun's luminosity. While the solar core produces electron neutrinos due to the fusion reaction, the solar corona plasma where fusion does not occur produces antineutrinos. The neutrinos from the core will interact with the antineutrinos from the corona to annihilate each other to produce two photons just like electron-positron annihilation does, but of lower frequency because of the much lower mass of the two neutrinos. This annihilation process explains the deficit of solar electron neutrinos as has been experimentally observed.

\section{The Difference between Electron-Positron Induced Fusion and the Current Inertial Fusion Experiments}

The electron-positron fusion reaction [2] is based on an experimental observation that has already been carried out whereby the fission energy from a nuclear bomb is used to set off the fusion (hydrogen) bomb as the one that was dropped by the United States above Namu Island in Bikini Atoll in the Pacific.

Inertial confinement fusion: magnetic, laser, and z-pinch fusion are all based on the process that takes place in the sun according to the Lawson criterion. Once a critical ignition temperature equal to that at the center of the Sun for fusion has been achieved, it must be maintained at that temperature for a long enough confinement time $(\mathrm{t})$ at high enough ion density $(\mathrm{n})$ to obtain a net yield for fusion:

$$
\begin{aligned}
& \text { nt } \geq 10^{14} \mathrm{~s} / \mathrm{cm}^{3} \quad \text { deuterium-tritium fusion } \\
& \mathrm{nt} \geq 10^{16} \mathrm{~s} / \mathrm{cm}^{3} \quad \text { deuterium-deuterium fusion }
\end{aligned}
$$

These inertial confinement experiments have proven to be unsuccessful so far to produce net energy gain because they do not require the high temperatures needed to start the fusion reaction as in the case of the fusion (hydrogen) bomb which is the same principle used in electron-positron induced fusion where 138 $\mathrm{kJ}$ of energy can deliver a temperature of 10 to the power $28 \mathrm{~K}$ to the D-T target at the center of the reactor, while the core of the sun has a temperature of only 27 Million K.

\section{Conflicts of Interest}

The author declares no conflicts of interest regarding the publication of this paper.

\section{References}

[1] Suplee, C. (2011) The Plasma Universe. CAMBRIDGE University Press, Cambridge, P. 54 .

[2] Irani, A. (2021) A Prototype Electron-Positron Fusion Reactor. Journal of High Energy Physics, Gravitation and Cosmology, 7, 949-951. 